\title{
Case Report: A Low-grade Uterine Leiomyosarcoma Showing Multiple Genetic Aberrations Including a Bi-allelic Loss of the Retinoblastoma Gene Locus, as well as Germ-line Uniparental Disomy for Part of the Long Arm of Chromosome 22
}

\author{
CARSTEN HOLZMANN ${ }^{1}$, DIRK KOCZAN ${ }^{2}$, THOMAS LOENING ${ }^{3}$, \\ BIRGIT ROMMEL $^{4}$ and JOERN BULLERDIEK ${ }^{1,4}$ \\ ${ }^{1}$ Institute of Medical Genetics, University Rostock Medical Center, Rostock, Germany; \\ ${ }^{2}$ Institute of Immunology, University Rostock Medical Center, Rostock, Germany; \\ ${ }^{3}$ Gerhard-Seifert-Referenzzentrum, Hamburg, Germany; \\ ${ }^{4}$ Human Genetics, University of Bremen, Bremen, Germany
}

\begin{abstract}
Background: Uterine leiomyosarcomas are rare tumors with adverse prognosis. Recently, it has been suggested that a possible genetic subgroup of these tumors might be characterized by bi-allelic deletions of the RBI locus. Here we report another uterine leiomyosarcoma with bi-allelic deletion of RB1 along with other genetic alterations. Case Report: A 52-year-old patient was admitted to the hospital for surgical removal of a polyp-like lesion in the uterine cavity. Histological examination revealed a grade 1 leiomyosarcoma with atypical mitoses and areas corresponding to a leiomyoma with bizarre nuclei. Results and Conclusion: This is the third case of a uterine leiomyosarcoma revealing bi-allelic RBI deletions. Thus, in the absence of monosomy 14 and/or mutations of MED12, this genetic alteration seems, indeed, to constitute a separate entity of these tumors. Histological analysis of the tumor along with its genetic intratumoral heterogeneity suggests its origin to be from a leiomyoma with bizarre nuclei. Furthermore, of considerable interest in the case presented here, is the identification of a large segment of chromosome 22 showing uniparental disomy. Along with the case presented here, recent data show that a genetic classification of all uterine leiomyosarcomas is recommended to reveal more information about clinical correlations of their different genetic subtypes. Due to array-based methods these
\end{abstract}

Correspondence to: Joern Bullerdiek, Institute of Medical Genetics, University Rostock Medical Center, Rostock, Germany. Tel: +49 3814947095, Fax: +49 3814947097, e-mail: joern.bullerdiek@ med.uni-rostock.de

Key Words: Uterine leiomyosarcoma, genetic alterations, $R B 1$, uniparental disomy, UPD, loss of heterozygosity, LOH, deletion. analyses can be well-carried out using paraffin-embedded samples.

Uterine leiomyosarcomas (ULMS) are rare smooth muscle malignancies associated with a bad prognosis. While most ULMS are thought to develop de novo, the question arises if those alterations also recurrently seen in uterine leiomyomas (UL) as well as in smooth muscle tumors of uncertain malignant potential (STUMP) suggest their origin from pre-existing UL. Here, we report one such case characterized by several genomic imbalances. However, while these imbalances as well as their intratumoral heterogeneity support the histological classification as a leiomyosarcoma, the simultaneous occurrence of areas corresponding to a leiomyoma with bizarre nuclei (ICD-O: $8893 / 0$ ) is speaking in favor of a lesion that has arisen from a pre-existing leiomyoma variant. Of note, the possibility of a UL-ULMS transition recently has been discussed by LieglAtzwanger et al. (1) based on their comparison between UL with bizarre nuclei and leiomyosarcomas suggesting that UL with bizarre nuclei constitute a separate genetic entity distinguishable from ordinary UL.

\section{Materials and Methods}

Tumor samples. After surgery, a polypous tumor presumably of uterine smooth muscle origin was fixed in paraformaldehyde $(4 \%$ in PBS), and processed for paraffin embedding. Tissue sections (1$2 \mu \mathrm{m})$ were de-paraffinized in xylene, rehydrated through a series of ethanol, and stained with hematoxylin/eosin (H\&E) for histological examination. DNA from the FFPE samples was isolated using the QIAamp DNA Mini Kit (Qiagen, Hilden, Germany) on a QIACube (Qiagen), according to the manufacturer's instructions. The amount of double-stranded DNA was measured using the Qubit dsDNA HS Assay Kit and a Qubit Fluorometer (Life Technologies, 


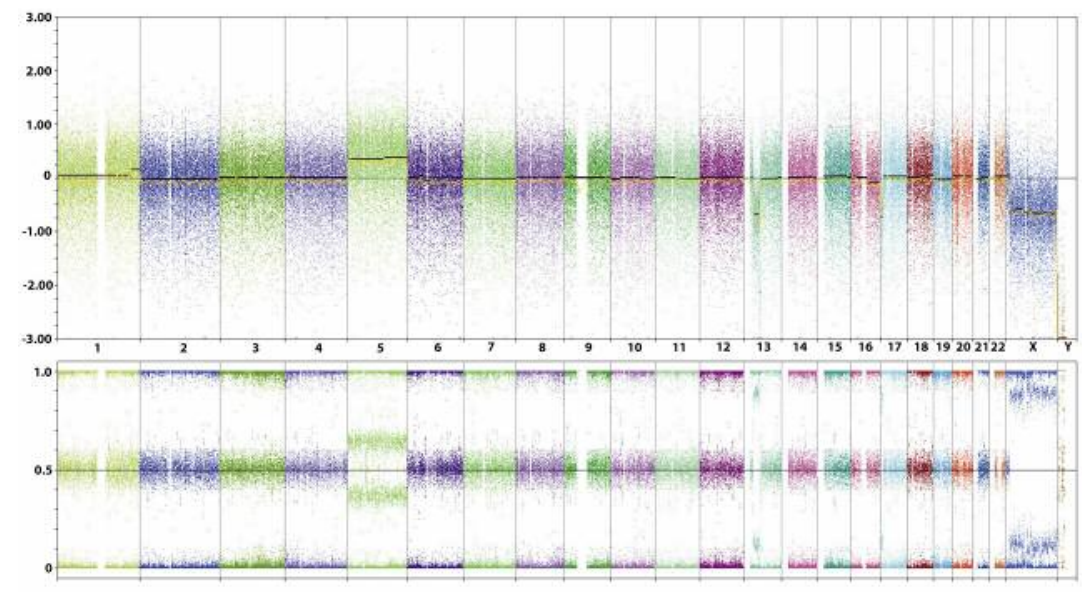

Figure 1. Genomic overview of the CNV array results for the low-grade uterine leiomyosarcoma showing losses of large segments of chromosomes 13, 17 , and the X-chromosome as well as the gain of the entire chromosome 5 as well as a copy number-neutral uniparental disomy for part of the long arm of chromosome 22. Top panel gives the copy number probe intensity calls and the bottom panel displays the calculation of B-allele frequencies (BAF).

Carlsbad, CA, USA). Analyses for mutations of MED12 and MIP array hybridization were performed as described recently $(2,3)$.

\section{Results}

Macroscopically, the tumor resected by hysteroscopy presented as a polypoid-like lesion that had protruded to the uterine cavity. Histological examination revealed a smooth muscle tumor with nuclear atypia, a moderately increased mitotic activity (8/10 high power fields) with some atypical mitoses, and necrosis characterized by abrupt transition from viable to non-viable areas. Accordingly, the tumor was classified as grade 1 leiomyosarcoma in combination with a leiomyoma with bizarre nuclei.

DNA from an FFPE-sample of the tumor was isolated and used for array analysis. By this analysis, the presence of several genomic regions displaying genomic imbalances were detected (Figure 1). Of these, deletions of the long arm of chromosome 13 (13q12.3-13q14.3), of the short arm of chromosome 17 (17p13.1-17pter) including the TP53 gene, and of almost the entire X-chromosome (Xp22.2-Xqter) occurred at apparently the same frequency of about $90 \%$ in the sample. Of note, the deletion of $17 \mathrm{p}$ was interspersed by a short region of approximately $0.320 \mathrm{Mb}$ (chr17p13: 1.574.342-1.896.930) with a copy number gain (Figure 2a). At a much lower frequency of roughly $50 \%$, trisomy 5 was noted speaking in favor for its secondary nature.

Analysis in more detail revealed two other remarkable findings: Besides the large deletion of 13q, a small, bi-allelic deletion encompassing the $R B 1$ locus was noted (Figure $2 \mathrm{~b}$ ). Furthermore, the tumor presented with an area of roughly $10 \mathrm{Mb}$ covering approximately one third of the long arm of chromosome 22 (22q12.1-22q13.1) displaying a normal copy number with uniparental disomy for both alleles. Apparently, this latter finding was present in all cells of the tumor suggesting its germ-line origin.

\section{Discussion}

Herein, we report a uterine leiomyosarcoma with losses of $R B 1$ and TP53 and an apparently germ-line UPD of part of chromosome 22. Alterations of all three loci have been described in ULMS and, except for the loss of the TP53 locus, also seem to occur in UL. However, the type and combination of these markers has not been reported previously.

Large, cytogenetically-visible deletions of the long arm of chromosome 13 are a rare, but recurrent finding in UL (4-6) and they have been repeatedly reported also in leiomyosarcomas (Table I) (7-13). Thus, the question arises if monoallelic deletion of the $R B 1$ locus is compatible with leiomyomatous growth while subsequent deletion of the second allele triggers malignant transformation. This model would perfectly fit with Knudson's original two-hit hypothesis for retinoblastoma growth (18) which has been later modified concerning the widespread occurrence of $R B 1$ inactivation in tumorigenesis. The question if bi-allelic inactivation in some instances is still compatible with benign growth has been addressed as well as possible consequences of monoallelic alterations of tumor suppressor genes $(19,20)$. As for ULMS, recently two other cases of LMS have been described where, akin to the case presented here, large deletions of $13 \mathrm{q}$ co-occurred with a much smaller deletions spanning the $R B 1$ locus (3). Interestingly, evidence for deletions of $R B 1$ in ULMS comes also from an older study applying Southern hybridization (21). In a study investigating leiomyoma-like areas in six cases of ULMS (10), the leiomyoma-like and uterine leiomyosarcoma areas were examined separately using high- 



Figure 2. In-depth analyses of abnormalities of chromosomes 13,17, and 22, respectively depict a bi-allelic loss of the RB1 locus, a deletion of $17 p$, and an uniparental disomy for part of the long arm of chromosome 22. A: CNV array results for part of the short arm of chromosome 17 showing a deletion encompassing the TP53 locus interspersed by a short segment revealing a copy number gain. Top panel gives the copy number probe intensity calls and the bottom panel displays the calculation of BAF. B: CNV array results for part of the long of chromosome 13 showing bi-allelic deletion of the RB1 locus. The panel gives the copy number probe intensity calls. C: CNV array results showing uniparental disomy for part of the long arm of chromosome 22. Top panel gives the copy number probe intensity calls and the bottom panel displays the calculation of $B A F$. Apparently, this UPD seems to occur in non-neoplastic cells as well because, unlikely the other aberrations, it is not restricted to part of the cells only (Figure 1). 
Table I. Frequent chromosomal deletions in uterine leiomyosarcomas.

\begin{tabular}{|c|c|c|c|}
\hline Affected chromosome & Deleted segments & Methods & Reference \\
\hline 1 & Deletions of the short arm encompassing varying segments & $\mathrm{CC} ; \mathrm{AGH}$ & $(3,13,14)$ \\
\hline 2 & Deletions of the short and long arm encompassing varying segments & $\mathrm{AGH}$ & $(5,13)$ \\
\hline 3 & Deletions of the long arm encompassing varying segments & $\mathrm{AGH}$ & (13) \\
\hline 4 & Deletions of the long arm encompassing varying segments & $\mathrm{AGH}$ & (13) \\
\hline 6 & Deletions of the short and long arm encompassing varying segments & $\mathrm{AGH}$ & (13) \\
\hline 8 & Deletions of the entire short arm & $\mathrm{AGH}$ & (13) \\
\hline 10 & Deletions of the short and long arm encompassing varying segments & $\mathrm{LOH} ; \mathrm{AGH}$ & $(5,13,15)$ \\
\hline 11 & Deletions of the short and long arm encompassing varying segments & $\mathrm{CC} ; \mathrm{AGH}$ & $(13,14)$ \\
\hline 12 & Deletions of the short arm encompassing varying segments & & $(5)$ \\
\hline 13 & $\begin{array}{l}\text { Deletions of the long arm encompassing varying segments often } \\
\text { including the } R B 1 \text { locus }\end{array}$ & $\mathrm{CC} ; \mathrm{AGH} ; \mathrm{NGS}$ & $(3,5,13,16)$ \\
\hline 14 & Deletions of the long arm encompassing varying segments & $\mathrm{AGH}$ & $(3,13,17)$ \\
\hline 15 & Deletions of the long arm encompassing varying segments & $\mathrm{AGH}$ & (13) \\
\hline 16 & Deletions of the long arm encompassing varying segments & AGH; & $(3,5)$ \\
\hline 19 & Deletions of the entire short arm & AGH & (13) \\
\hline 22 & Deletions of the entire long arm or encompassing varying segments & AGH & $(3,13,17)$ \\
\hline $\mathrm{X}$ & Deletions of the short and long arm encompassing varying segments & $\mathrm{AGH}$ & (13) \\
\hline
\end{tabular}

CC: Classical cytogenetics, AGH: Array comparative genomic hybridization, NGS: Next generation sequencing, TES: targeted exome sequencing, LOH: Microsatellite analysis for loss of heterozygosity.

density oligonucleotide array-CGH to compare the genetic abnormalities occurring in both areas. While the "benign areas" shared nearly all abnormalities with their corresponding malignant parts, a couple of genomic alterations were restricted to "malignant areas" only. In two of these cases losses of $13 q$ including the $R B 1$ locus were confined to the sarcoma areas but no bi-allelic losses were noted. However, to the best of our knowledge, this is the third ULMS with such a bi-allelic alteration of the RBI locus shown by array analysis and may suggest the existence of a subgroup of ULMS driven by biallelic losses of the $R B 1$ locus and lacking MED12 mutations.

In contrast to $13 \mathrm{q}$ deletions, deletions of the short arm of chromosome 17 including the TP53 locus are not known as recurrent chromosomal aberrations in UL. Of note, a deletion of the short arm of chromosome 17 encompassing the TP53 locus present in the ULMS, but not the UL area, has been found in one of six cases studied by Mittal et al. by array hybridization (10). Moreover, loss of heterozygosity for TP53 as well as its mutations has been described in ULMS repeatedly $(8,22)$.

Albeit at a low frequency, monosomy 22 or partial deletions of $22 q$, like deletions of $13 q$, are also recurrently found in UL $(6,23)$. Also, in a genome-wide association study a locus assigned to $22 q 13.1$ was among three loci that revealed significant association with uterine fibroids (24). Quite recently, $D E P D C 5$ has been considered being a possible target gene (25). In the present case this locus is apparently affected by germline UPD. For some chromosomes and their regions, respectively, uniparental disomies are known not to coincide with any adverse phenotypic effects for their carriers (26). Uniparental disomy for the entire long arm of chromosome 22 resulting from a balanced Robertsonian translocation between both long arms of chromosome 22 has been described in a 25 year-old normal healthy male (27). Likewise, other cases of maternal transmission of a $\mathrm{t}(22 \mathrm{q} ; 22 \mathrm{q})$ translocation have been reported not to coincide with phenotypic effects $(28,29)$. In contrast, in one case maternal UPD for the entire chromosome 22 likely resulting from monosomy rescue (30) was associated with neurologic (developmental delay, speech delay, autism, or intellectual disability) disease phenotype in a four year old boy. Nevertheless, in general UPD for even the entire $22 q$ does not seem necessarily to affect the phenotype.

In general, the combination of ULMS with areas suggesting a pre-existing UL has been reported in single cases (31), as well as in series of ULMS. Of eleven ULMS studied for intratumoral morphologic heterogeneity Mittal and Joutovsky (32) had been able to identify morphologically benign tumor areas in five tumors. In a larger study by the same group, 18/26 ULMS histologically displayed associated leiomyoma-like areas (10). In the case presented herein histopathological heterogeneity is associated with genetic alterations occurring at a varying percentage of the tumor cell population. This suggests the existence of RBI-two hit tumors as a possible subgroup of ULMS. The presence of a large germline UPD covering the DEPDC5 locus raises the question if certain UPDs may be factors triggering the rare leiomyomaleiomyosarcoma transition.

\section{References}

1 Liegl-Atzwanger B, Heitzer E, Flicker K, Müller S, Ulz P, Saglam O, Tavassoli F, Devouassoux-Shisheboran M, Geigl J and Moinfar F: Exploring chromosomal abnormalities and genetic changes in uterine smooth muscle tumors. Mod Pathol 29: 1-16, 2016. 
2 Markowski DN, Bartnitzke S, Löning T, Drieschner N, Helmke $\mathrm{BM}$ and Bullerdiek J: MED12 mutations in uterine fibroids their relationship to cytogenetic subgroups. Int J Cancer 131: 1528-1536, 2012.

3 Holzmann C, Markowski DN, von Leffern I, Löning T and Bullerdiek J: Patterns of chromosomal abnormalities that can improve diagnosis of uterine smooth muscle tumors. Anticancer Res 35: 6445-6456, 2015.

4 Meloni AM, Surti U and Sandberg AA: Deletion of chromosome 13 in leiomyomas of the uterus. Cancer Genet Cytogenet 53: 199-203, 1991.

$5 \mathrm{Hu}$ J, Khanna V, Jones M and Surti U: Genomic alterations in uterine leiomyosarcomas: Potential markers for clinical diagnosis and prognosis. Genes Chromosom Cancer 31: 117-124, 2001

6 Sandberg AA: Updates on the cytogenetics and molecular genetics of bone and soft tissue tumors: leiomyoma. Cancer Genet Cytogenet 158: 1-26, 2005.

7 Cho YL, Bae S, Koo MS, Kim KM, Chun H-J, Kim CK, Ro DY, Kim JH, Lee C-H, Kim Y-W and Ahn WS: Array comparative genomic hybridization analysis of uterine leiomyosarcoma. Gynecol Oncol 99: 545-551, 2005.

8 Zhai YL, Nikaido T, Orii A, Horiuchi A, Toki T and Fujii S: Frequent occurrence of loss of heterozygosity among tumor suppressor genes in uterine leiomyosarcoma. Gynecol Oncol 75: 453-459, 1999.

9 Raish M, Khurshid M, Ansari MA, Chaturvedi PK, Bae S-M, Kim JH, Park EK, Park DC and Ahn WS: Analysis of molecular cytogenetic alterations in uterine leiomyosarcoma by array-based comparative genomic hybridization. J Cancer Res Clin Oncol 138: 1173-1186, 2012.

10 Mittal KR, Chen F, Wei JJ, Rijhvani K, Kurvathi R, Streck D, Dermody $\mathrm{J}$ and Toruner GA: Molecular and immunohistochemical evidence for the origin of uterine leiomyosarcomas from associated leiomyoma and symplastic leiomyoma-like areas. Mod Pathol 22: 1303-1311, 2009.

11 Otaño-Joos M, Mechtersheimer G, Ohl S, Lehnert T, Willeke F, Möller P, Otto HF, Lichter P and Joos S: Analysis of chromosome copy number changes in leiomyosarcoma through molecular cytogenetic methods. Verh Dtsch Ges Pathol 82: 207-209, 1998.

12 Derré J, Lagacé R, Nicolas A, Mairal A, Chibon F, Coindre JM, Terrier P, Sastre $\mathrm{X}$ and Aurias A: Leiomyosarcomas and most malignant fibrous histiocytomas share very similar comparative genomic hybridization imbalances: an analysis of a series of 27 leiomyosarcomas. Lab Investig 81: 211-215, 2001.

13 Croce S, Ribeiro A, Brulard C, Noel J-C, Amant F, Stoeckle E, Devouassoux-Shisheborah M, Floquet A, Arnould L, Guyon F, Mishellany F, Garbay D, Cuppens T, Zikan M, Leroux A, Frouin E, Duvillard P, Terrier P, Farre I, Valo I, Macgrogan GM and Chibon F: Uterine smooth muscle tumor analysis by comparative genomic hybridization: a useful diagnostic tool in challenging lesions. Mod Pathol 28: 1001-1010, 2015.

14 Laxman R, Currie JL, Kurman RJ, Dudzinski M and Griffin CA: Cytogenetic profile of uterine sarcomas. Cancer 71: 1283-1288, 1993.

15 Quade BJ, Pinto AP, Howard DR, Peters WA and Crum CP: Frequent loss of heterozygosity for chromosome 10 in uterine leiomyosarcoma in contrast to leiomyoma. Am J Pathol 154: 945-950, 1999.

16 Lee PJ, Yoo NS, Hagemann IS, Pfeifer JD, Cottrell CE, Abel HJ and Duncavage EJ: Spectrum of Mutations in Leiomyosarcomas Identified By Clinical Targeted Next-Generation Sequencing. Exp Mol Pathol 102: 156-161, 2017.
17 Levy B, Mukherjee T and Hirschhorn K: Molecular cytogenetic analysis of uterine leiomyoma and leiomyosarcoma by comparative genomic hybridization. Cancer Genet Cytogenet 121: 1-8, 2000.

18 Knudson AG: Mutation and cancer: statistical study of retinoblastoma. Proc Natl Acad Sci USA 68: 820-823, 1971.

19 Knudson AG: Hereditary cancer: Two hits revisited. J Cancer Res Clin Oncol 122: 135-140, 1996.

20 Berger AH, Knudson AG and Pandolfi PP: A continuum model for tumour suppression. Nature 476: 163-169, 2011.

21 Stratton MR, Williams S, Fisher C, Ball A, Westbury G, Gusterson BA, Fletcher CD, Knight JC, Fung YK and Reeves BR: Structural alterations of the RB1 gene in human soft tissue tumours. Br J Cancer 60: 202-205, 1989.

22 Yang CY, Liau JY, Huang WJ, Chang YT, Chang MC, Lee JC, Tsai JH, Su YN, Hung CC and Jeng YM: Targeted next-generation sequencing of cancer genes identified frequent TP53 and ATRX mutations in leiomyosarcoma. Am J Transl Res 7: 2072-2081, 2015.

23 Vanni R, Lecca U and Faa G: Uterine leiomyoma cytogenetics. II. Report of forty cases. Cancer Genet Cytogenet 53: 247-256, 1991.

24 Cha P-C, Takahashi A, Hosono N, Low S-K, Kamatani N, Kubo $\mathrm{M}$ and Nakamura Y: A genome-wide association study identifies three loci associated with susceptibility to uterine fibroids. Nat Genet 43: 447-450, 2011.

25 Mehine M, Heinonen HR, Sarvilinna N, Pitkänen E, Mäkinen N, Katainen R, Tuupanen S, Bützow R, Sjöberg J and Aaltonen LA: Clonally related uterine leiomyomas are common and display branched tumor evolution. Hum Mol Genet 24: 4407-4416, 2015.

26 Liehr T: Cases with uniparental disomy, 2017. Available from: http://upd-tl.com/upd.html [last accessed January 13, 2017].

27 Schinzel AA, Basaran S, Bernasconi F, Karaman B, Yukselapak $M$ and Robinson WP: Maternal uniparental disomy-22 has no impact on the phenotype. Am J Hum Genet 54: 21-24, 1994.

28 Kirkels VGHJ, Hustinx WJ and Scheres JMJC: Habitual abortion and translocation (22q;22q): unexpected transmission from a mother to her phenotypically normal daughter. Clin Genet 18: 456-461, 1980.

29 Chopade DK, Harde H, Ugale P and Chopade S: Unexpected Inheritance of a Balanced Homologous translocation $t(22 q ; 22 q)$ from father to a phenotypically normal daughter. Indian J Hum Genet 20: 85-88, 2014.

30 Yang Y, Muzny DM, Xia F, Niu Z, Person R, Ding Y, Ward P, Braxton A, Wang M, Buhay C, Veeraraghavan N, Hawes A, Chiang T, Leduc M, Beuten J, Zhang J, He W, Scull J, Willis A, Landsverk M, Craigen WJ, Bekheirnia MR, Stray-Pedersen A, Liu P, Wen S, Alcaraz W, Cui H, Walkiewicz M, Reid J, Bainbridge M, Patel A, Boerwinkle E, Beaudet AL, Lupski JR, Plon SE, Gibbs $\mathrm{R}$ and Eng CM: Molecular findings among patients referred for clinical whole-exome sequencing. Jama 312: 1870, 2014.

31 Mittal K, Popiolek D and Demopoulos RI: Uterine myxoid leiomyosarcoma within a leiomyoma. Hum Pathol 31: 398-400, 2000.

32 Mittal K and Joutovsky A: Areas with benign morphologic and immunohistochemical features are associated with some uterine leiomyosarcomas. Gynecol Oncol 104: 362-365, 2007.

Received February 10, 2017

Revised March 20, 2017

Accepted March 27, 2017 